\title{
Avaliação epidemiológica da Síndrome Hipertensiva Específica da Gravidez em um município da região norte do Brasil
}

Epidemiological evaluation of specific hypertensive syndrome of pregnancy in a municipality in the north region of brazil

Evaluación epidemiológica del síndrome hipertensivo específico del embarazo en un municipio de la región norte de Brasil

Recebido: 08/04/2021 | Revisado: 16/04/2021 | Aceito: 18/04/2021 | Publicado: 28/04/2021

Hannah Imbelloni Evangelista ORCID: https://orcid.org/0000-0003-0822-4298

Universidade Federal do Pará, Brasil

E-mail: hannah.imbelloni@yahoo.com.br

Talita Pompeu da Silva ORCID: https://orcid.org/0000-0001-7450-8454

Universidade Federal do Maranhão, Brasil E-mail: tpsmedicina@hotmail.com

Gabriel Aguiar dos Santos

ORCID: https://orcid.org/0000-0002-0635-266X Universidade do Estado do Pará, Brasil E-mail: gab.ads96@gmail.com

Renan Corrêa da Costa Duarte ORCID: https://orcid.org/0000-0002-4820-2117 Universidade do Estado do Pará, Brasil E-mail: tpsmedicina@hotmail.com

Adriani Ferreira do Carmo ORCID: https://orcid.org/0000-0003-4405-3529 Universidade Potiguar, Brasil E-mail: adriani.carmo@gmail.com

Marcio Lima da Costa

ORCID: https://orcid.org/0000-0002-3717-5483 Universidade Federal do Pará, Brasil E-mail: marcio_limac@hotmail.com Andrew Silva Matos ORCID: https://orcid.org/0000-0003-3071-8745 Universidade Federal do Pará, Brasil E-mail: andrewmatos20@gmail.com

Davis Wilker Nascimento Vaz

ORCID: https://orcid.org/0000-0002-0601-8194 Universidade do Estado do Pará, Brasil E-mail: daviswilkervaz@gmail.com

\section{Resumo}

Objetivo: avaliar os aspectos epidemiológicos da Síndrome Hipertensiva Específica da Gravidez (SHEG), em Belém, Pará, durante o período de janeiro de 2016 até dezembro de 2020. Metodologia: estudo epidemiológico, retrospectivo e quantitativo cujo dados foram obtidos mediante informações disponíveis no Departamento de Informática do Sistema de Saúde Unificado (DATASUS). Foram escolhidas as seguintes variáveis epidemiológicas: número de internações, anos das internações (2016 a 2020), faixa etária, etnia (branca, preta, parda, amarela e sem informação) e número de óbitos por ano. Resultados: O estudo identificou um total de 7386 casos de SHEG nos 5 anos avaliados. A maior parte ocorreu em pacientes na faixa etária de 20 a 34 anos (66,42\%), 16,93\% das pacientes com SHEG eram adolescentes (10 a 19 anos) e 16,63\% possuíam idade igual ou superior a 35 anos. Com relação à etnia das participantes da pesquisa, houve predomínio da cor parda $(88,89 \%)$, seguida pela branca $(4,45 \%)$, preta $(1,81 \%)$ e amarela $(0,06 \%)$, respectivamente. O estudo também identificou um número considerável de óbitos ocasionados por SHEG. Conclusão: A presente pesquisa evidenciou a importância dos profissionais de saúde, principalmente da APS, sempre estarem atentos aos fatores de risco, para assim, identificar, previamente, aquelas gestantes com maior risco de evoluirem para a SHEG de modo a orientá-las e prover uma assistência pré-natal mais integral e particularizada. Ademais, é imperativo melhorias no manejo da gestante nas urgências e emergências obstétricas com o intuito de diminuir a mortalidade associada à SHEG.

Palavras-chave: Hipertensão Induzida pela gravidez; Pré-eclâmpsia; Eclampsia. 


\begin{abstract}
Objective: to evaluate the epidemiological aspects of Specific Hypertensive Syndrome of Pregnancy (SHEG), in Belém, Pará, during the period from January 2016 to December 2020. Methodology: epidemiological, retrospective and quantitative study whose data were obtained through information available at the Department Informatics of the Unified Health System (DATASUS). The following epidemiological variables were chosen: number of hospitalizations, years of hospitalization (2016 to 2020), age group, ethnicity (white, black, brown, yellow and without information) and number of deaths per year. Results: The study identified a total of 7386 cases of SHEG in the 5 years evaluated. Most of them occurred in patients aged 20 to 34 years $(66.42 \%), 16.93 \%$ of patients with SHEG were adolescents (10 to 19 years) and $16.63 \%$ were aged 35 or over . Regarding the ethnicity of the research participants, there was a predominance of brown color $(88.89 \%)$, followed by white $(4.45 \%)$, black $(1.81 \%)$ and yellow $(0.06 \%)$, respectively. The study also identified a considerable number of deaths caused by SHEG. Conclusion: The present research showed the importance of health professionals, mainly from PHC, to always be aware of risk factors, in order to identify, in advance, those pregnant women with a higher risk of evolving to SHEG in order to guide them and provide more comprehensive and individualized prenatal care. In addition, improvements in the management of pregnant women in urgent and obstetric emergencies are imperative in order to reduce mortality associated with SHEG.
\end{abstract}

Keywords: Hypertension, Pregnancy-induced; Pre-eclampsia; Eclampsia.

\title{
Resumen
}

Objetivo: evaluar los aspectos epidemiológicos del Síndrome Hipertensivo Específico del Embarazo (SHEG), en Belém, Pará, durante el período de enero de 2016 a diciembre de 2020. Metodología: estudio epidemiológico, retrospectivo y cuantitativo cuyos datos se obtuvieron a través de la información disponible en el Departamento. Informática del Sistema Único de Salud (DATASUS). Se eligieron las siguientes variables epidemiológicas: número de hospitalizaciones, años de hospitalización (2016 a 2020), grupo de edad, etnia (blanca, negra, parda, amarilla y sin información) y número de muertes por año. Resultados: El estudio identificó un total de 7386 casos de SHEG en los 5 años evaluados. La mayoría de ellos ocurrieron en pacientes de 20 a 34 años $(66,42 \%$ ), el 16,93\% de los pacientes con SHEG eran adolescentes (10 a 19 años) y el 16,63\% tenían 35 años o más. En cuanto a la etnia de los participantes de la investigación, hubo predominio del color marrón $(88,89 \%)$, seguido del blanco $(4,45 \%)$, negro $(1,81 \%)$ y amarillo $(0,06 \%)$, respectivamente. El estudio también identificó un número considerable de muertes causadas por SHEG. Conclusión: La presente investigación mostró la importancia de que los profesionales de la salud, principalmente de la APS, estén siempre al tanto de los factores de riesgo, con el fin de identificar, con anticipación, a aquellas gestantes con mayor riesgo de evolucionar a SHEG con el fin de orientarlas y brindarles atención prenatal más integral e individualizada. Además, las mejoras en el manejo de las mujeres embarazadas en emergencias urgentes y obstétricas son imperativas para reducir la mortalidad asociada con SHEG.

Palabras clave: Hipertensión inducida en el embarazo; Preeclampsia; Eclampsia.

\section{Introdução}

O período gestacional é caracterizado por importantes mudanças tanto fisiológicas quanto sociais e emocionais para a mulher. Contudo, pode cursar com grandes complicações. A Sindrome Hipertensiva Específica da Gravidez (SHEG) é uma das principais, sendo a primeira causa de morte materna no Brasil e a segunda no mundo, perdendo apenas para as hemorragias (Fassarella et al., 2020; Ferreira et al., 2021 Nunes et al., 2020).

Devido ao alto índice de morbimortalidade relacionada à SHEG, é fundamental que se busque identificar as gestações com maior risco de evolução para essa síndrome. Dentre os principais fatores de risco associados ao desenvolvimento de SHEG têm-se: a obesidade, idade materna avançada, diabetes mellitus, hipertensão arterial, histórico pessoal e/ou familiar de SHEG, inatividade física e hábitos alimentares inapropriados (Araújo et al., 2017; Soares et al., 2019).

Nesse sentido, a Atenção Primária a Saúde (APS) apresenta um papel primordial. Nesse nível de atenção é possível a criação de um vínculo mais forte da gestante com a equipe multiprofissional de modo a prevenir, diagnosticar e tratar doenças precocemente. Portanto, o ratreio e prevenção da SHEG deve ser uma das prioridades da APS desde a primeira consulta da assistência pré-natal (Ferreira et al., 2017; Neto et al., 2021).

A SHEG pode ser classificada em: Hipertensão Gestacional (gestante previamente não hipertensa com diagnóstico de hipertensão após a $20^{\mathrm{a}}$ semana de gestação), Hipertensão Crônica (hipertensão prévia a gestação ou diagnosticada até a $20^{\mathrm{a}}$ semana de gestação), Pré-Eclâmpsia (hipertensão diagnosticada após 20 semanas de gestação associada à proteinúria $>300$ mg/24 horas), Pré-Eclâmpsia sobreposta à Hipertensão Crônica (gestante previamente hipertensa que evolui com pré eclâmpsia na gestação), Eclâmpsia (presença de convulsão, não atribuída a outras causas, em mulheres com Pré-eclâmpsia) (Oliveira et 
al., 2006; Bastos et al., 2021; Neto et al., 2021).

A presença de SHEG eleva consideravelmente o risco de surgimento de importantes complicações tanto maternas quanto fetais como o abortamento, restrição de crescimento intrauterino, prematuridade, óbito intrauterino, sofrimento fetal e baixo peso ao nascimento. Uma das afecções mais temidas é a denominada Síndrome HELLP caracterizada pela elevação de enzimas hepáticas, plaquetopenia e hemólise que pode evoluir com importantes complicações como insuficiência renal, edema agudo de pulmão, coagulação intravascular disseminada (CIVD), elevando assim, risco de morte (Sousa et al., 2020; Fiorio et al., 2020, Ribeiro et al., 2020).

Nessa perspectiva, o objetivo do presente estudo foi avaliar os aspectos epidemiológicos da SHEG, no Município de Belém, Estado do Pará, Brasil, durante o período de janeiro de 2016 até dezembro de 2020.

\section{Metodologia}

Caracteriza-se como um estudo epidemiológico, retrospectivo e quantitativo cujo dados foram obtidos mediante informações disponíveis no Departamento de Informática do Sistema de Saúde Unificado (DATASUS), do Ministério da Saúde, um importante departamento dotado de sistemas de saúde que disponibilizam informações úteis tanto para avaliação quanto para a tomada de decisões em saúde (Piccolo, 2018).

O estudo foi realizado no município de Belém, no Estado do Pará, localizado na região norte do Brasil. A população do estudo contituiu-se de pacientes com SHEG que foram internadas durante o período de janeiro de 2016 a dezembro de 2020. As participantes foram divididas em 3 faixas etárias: 10 a 19 anos, 20 a 34 anos e 35 anos ou mais. Foram escolhidas as seguintes variáveis epidemiológicas: número de internações, anos das internações (2016 a 2020), faixa etária, etnia (branca, preta, parda, amarela e sem informação) e número de óbitos por ano.

Após a coleta, os dados foram organizados de forma gráfica e analisados estatísticamente através da utilização dos sofwares Excel 2013 e Word 2013. Destaca-se que por se tratar de informações de domínio público, não foi necessária a avaliação pelo Comitê de Ética em Pesquisa.

\section{Resultados e Discussão}

O estudo identificou um total de 7386 casos de SHEG nos 5 anos avaliados (Figura 1), sendo de 1151, 1446, 1526, 1449 e 1814, nos anos 2016, 2017, 2018, 2019 e 2020, respectivamente. Em virtude do aumento da prevalência das Doenças Crônicas não Transmissíveis, com destaque para Hipertensão Arterial Sistêmica, tem ocorrido também o aumento do número dos casos de SHEG e outras complicações obstétricas nos últimos anos (Rech \& David, 2020; Sousa et al., 2020). 
Figura 1: Distribuição dos casos de SHEG durante o período de 2016 a 2020 em Belém, Pará.

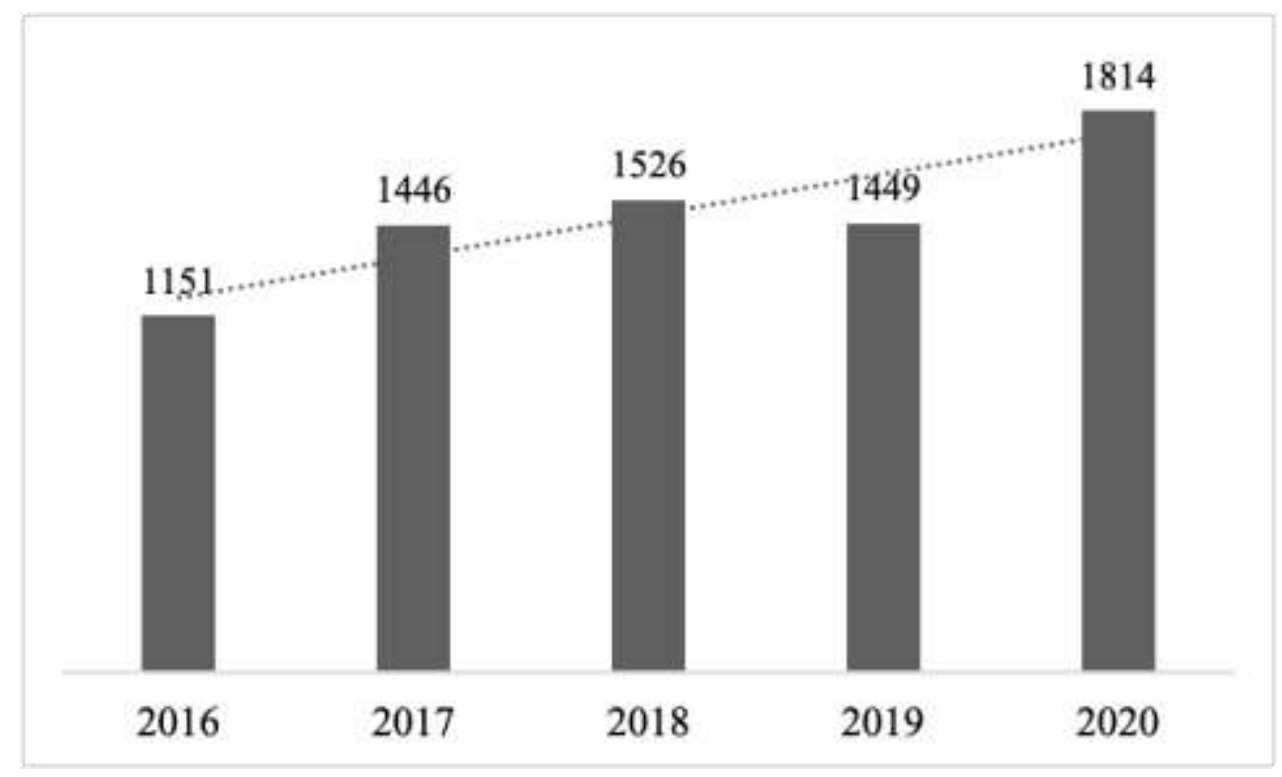

Fonte: Ministério da Saúde. SIH/SUS, (2021).

Com relação à faixa etária (Figura 2), observou-se que a maior parte dos casos de SHEG ocorreu em pacientes com idade inferior a 35 anos (83,35\%). Sousa et al (2020), ao avaliar a epidemiologia da SHEG em um Hospital Público localizado em São Paulo, encontrou resultados semelhantes, com 62,3\% das gestantes com idade igual ou inferior a 35 anos. No estudo de Rodrigues et al. (2020), realizado no Município de Fortaleza, Ceará, 74,5\% das pacientes com SHEG possuíam menos que 30 anos.

Figura 2: Distribuição da faixa etária das pacientes com SHEG em Belém, Pará.

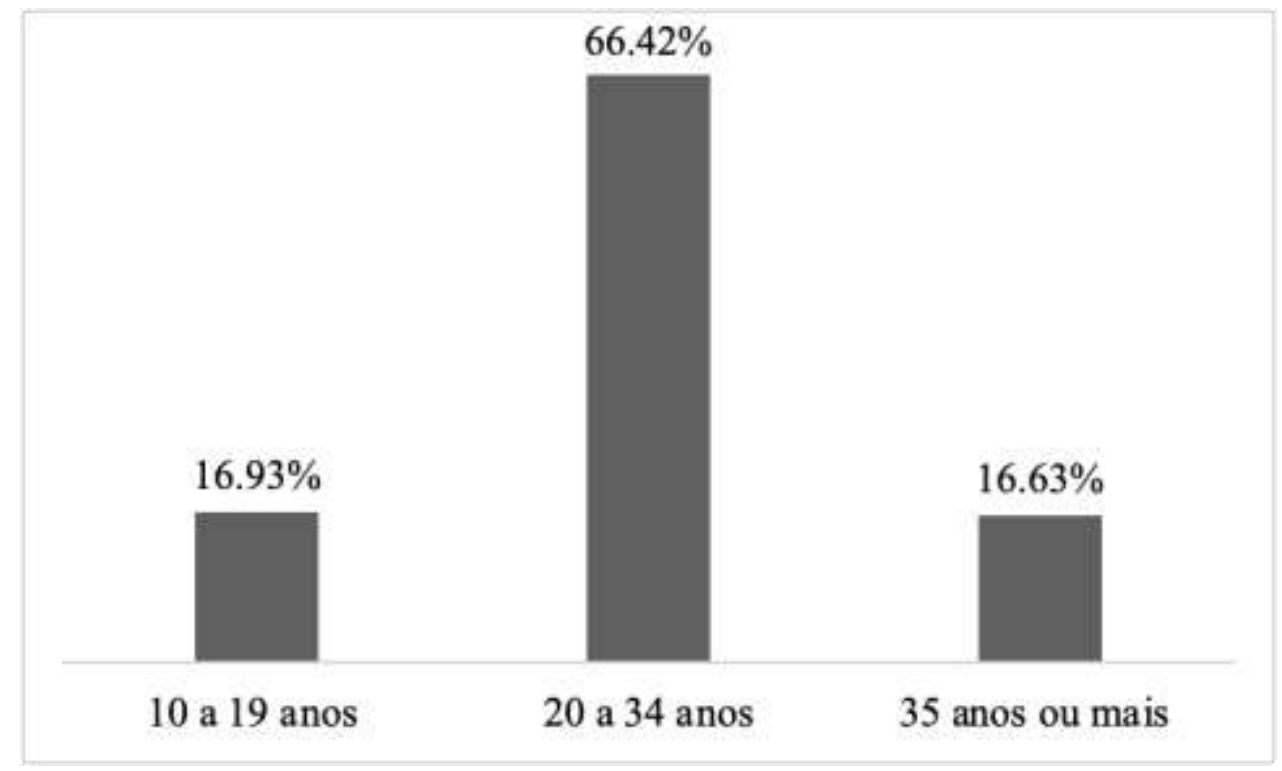

Fonte: Ministério da Saúde. SIH/SUS, (2021).

A Figura 2 também revela que 16,93\% dos pacientes com SHEG possuíam de 10 a 19 anos. Em virtude do início a cada vez mais precoce da vida sexual, tem aumentado o número de gestantes adolescentes no Brasil, principalmente nas classes menos privilegiadas socioeconômicamente. A gestação na adolescência é considerada um importante problema de 
saúde pública no mundo. Nessa faixa etária, a SHEG também é a complicação obstétrica mais comum (Ferreira et al., 2021; Alves et al., 2021).

A presente pesquisa também averigou que 16,63\% das pacientes com SHEG apresentavam 35 anos ou mais (Figura 2). Destaca-se que grávidas com idade igual ou superior a 35 anos são definidas como mulheres em idade materna avançada (IMA) e estão mais propensas ao aparecimento de complicações obstétricas tais como, restrição de crescimento intrauterino, diabetes mellitus gestacional, trabalho de parto prematuro, placenta prévia, descolamento prematuro de placenta e SHEG (Viellas et al., 2021; Barboza et al.,2020).

Com relação à etnia das participantes da pesquisa, a Tabela 1 mostra que houve predomínio da cor parda $(88,89 \%)$, seguida pela branca $(4,45 \%)$, preta $(1,81 \%)$ e amarela $(0,06 \%)$, respectivamente. No estudo de Rodrigues et al. (2020) realizado no estado do Ceará, foi observado que as pardas compuseram a maioria $(66,6 \%)$ da amostra das pacientes com diagnóstico de SHEG. Na pesquisa de Brito et al. (2015), realizado no município de João de Pessoa, também houve predomínio da cor parda sendo responsável por 57\% dos casos de SHEG.

Tabela 1: Distribuição da etnia/cor das pacientes com SHEG em Belém, Pará.

\begin{tabular}{|lll|}
\hline ETNIA/COR & NÚMERO & $\%$ \\
\hline Branca & 329 & $4,45 \%$ \\
\hline Preta & 134 & $1,81 \%$ \\
\hline Parda & 6566 & $88,89 \%$ \\
\hline Amarela & 5 & $0,06 \%$ \\
\hline Sem informação & 352 & $4,76 \%$ \\
\hline
\end{tabular}

Fonte: Ministério da Saúde. SIH/SUS, (2021).

O estudo também identificou o número de óbitos maternos (Tabela 2) que foram ocasionados por SHEG. A mortalidade materna é um importante indicador de qualidade de vida de uma população sendo a SHEG a principal causa de morte, tanto materna quanto fetal, nos países em desenvolvimento como o Brasil (Pereira et al., 2017; Silva \& Cismer, 2017).

Tabela 2: Óbitos ocasionados por SHEG no período de 2016 a 2020 em Belém, Pará.

\begin{tabular}{|lll|}
\hline ANO & N. ${ }^{\circ}$ DE ÓBITOS & $\%$ \\
\hline $\mathbf{2 0 1 6}$ & 1 & $6,66 \%$ \\
\hline $\mathbf{2 0 1 7}$ & 5 & $33,33 \%$ \\
\hline $\mathbf{2 0 1 8}$ & 3 & $20 \%$ \\
\hline $\mathbf{2 0 1 9}$ & 4 & $26,66 \%$ \\
\hline $\mathbf{2 0 2 0}$ & 2 & $13,3 \%$ \\
\hline
\end{tabular}

Fonte: Ministério da Saúde. SIH/SUS, (2021).

A literatura científica aponta que uma assistência pré-natal inadequada na APS aumenta o risco de complicações obstétricas como a SHEG e, consequentemente, a chance de morte. Ademais, o atendimento e manejo inadequado de parte considerável das gestantes com SHEG nas urgências e emergências, também contribuem fortemente para a elevação das taxas de mortalidade maternas e fetais (Cortinhas et al., 2019). 


\section{Conclusão}

O estudo identificou um aumento progressivo do número de casos de SHEG nos 5 anos avaliados. A maioria das pacientes possuíam idade entre 20 e 34 anos, contudo as demais faixas etárias também apresentaram uma prevalência relevante de pacientes com SHEG. Com relação à cor, a mais prevalente foi a parda. A pesquisa também observou uma taxa de óbitos considerável por essa etiologia.

O estudo evidenciou a importância dos profissionais de saúde, principalmente da APS, sempre estarem atentos aos fatores de risco, com o intuito de identificar, previamente, aquelas gestantes com maior risco de evoluirem para a SHEG de modo a orienta-las e prover uma assistência pré-natal mais integral e particularizada.

Faz-se necessário, também, melhorias no referenciamento da gestante com SHEG para o pré-natal de alto risco, quando necessário. Ademais, é imperativo melhorias no manejo da gestante com SHEG nas urgências e emergências obstétricas, através da melhor capacitação de profissionais da saúde e aumento de insumos hospitalares, com o intuito de melhorar o tratamento dessas pacientes e, por conseguinte, diminuir a mortalidade associada à SHEG.

\section{Referências}

Alves, R. S. S., Sousa, F. L. L., Leite, A. C., Silva, M. P. B., Silva, J. K. A., Silva, E. R., Santos, S. F., Rodrigues, H. A., Maia, C. C., Silvestre, F. E. R., Almeida, L. F., Sorio, C. F., Favalessa, A. R., Santos, B. K. O., Amando, M. A. O., Mendonça, L. C. J., Miranda, C. Q., Fernandes, J. M., \& Morais, L. S. F. (2021). Gravidez na adolescência: Contribuições dos profissionais de saúde frente à educação sexual e reprodutiva. Research, Society and Development, 10(2), e20010211282.

Araújo, I. F. M., Santos, P. A., Santos, P. A., \& Franklin, T. A. (2017). Síndromes hipertensivas e fatores de risco associados à gestação. Rev enferm UFPE on line, 11(Supl. 10), 4254-4262.

Barboza, B. P., Calil, C., Trigo, I. G. P. F., Eller, J. C., Silva, L. R., Vaz, M. R., \& Esteves, A. P. V. S. (2020). Idade materna avançada e seus desfechos. Revista Cadernos de Medicina, 2(3), 146-151.

Bastos, P. S., Alves, K. K. A. F., Silva, F. G., Pereira, W. D., Amorim, C. F., Carvalho, C. B., Cavalcante, C. M., Cavalcante, N. S. A., \& Oliveira, L. Q. (2021). Assistência de enfermagem a gestante com hipertensão na Atenção Básica: um relato de experiência. Research, Society and Development, 10(1), e55510112009.

Brito, K. K. G., Moura, J. R. P., Sousa, M. J., Brito, J. V., Oliveira, S. H. S., Soares, M. J. G. O. (2015). Prevalência das síndromes hipertensivas específicas da gestação (SHEG). J. res.: fundam. care. Online, 7(3), 2717-2725.

Cortinhas, A. B. B., Miranda, F. F. S., Toth, M. V. B., Freitas, R. F., Costa, T. R., \& Esteves, A. P. V. S. (2019). Pré- eclâmpsia e mortalidade materna. Revista Caderno de Medicina, 2(1), 63-73.

Fassarella, B. P. A., Almeida, G., Teles, D. A., Ortiz, L. S., Silva, I. S., Neves, K. C., Costa, P. A. F. S., Ribeiro, W. A., \& Evangelista, D. S. (2020). Cuidados de enfermagem direcionados à gestante portadora de doença hipertensiva específica da gravidez. Research, Society and Development, 9(9), e343996768.

Ferreira, J. P. N., Santos, L. D. L., Bacelar, A. P. A., Amaral, S. M., Gonçalves, L. N., Sousa, A. M. R., Torres, M. A. O., \& Martins, M. G. (2021). Síndromes hipertensivas específicas da gestação em adolescentes e suas repercussões maternas e perinatais: uma revisão integrativa de literatura. Brazilian Journal of Development, 7(3), 32204-32217.

Ferreira, S. S., Martins, A. C., Magalhães, A. C., \& Martins, H. (2017). Ácido acetilsalicílico na prevenção da pré-eclâmpsia: uma revisão baseada na evidência. Rev Port Med Geral Fam, 33, 118-132.

Fiorio, T. A., Pereira, T. M., Righi, M. G., Vieira, A. P., Follador, F. A. C., Wendt, G. W., \& Farreto, L. E. D. (2020). Doença hipertensiva específica da gestação: prevalência e fatores associados. Brazilian Journal of Development, 6(6), 35921-35934.

Neto, J. C., Santos, P. S. P., Feitosa, E. M. S., \& Oliveira, J. D. (2021). Guias de assistência à mulher com síndrome hipertensiva na Atenção Primária: revisão integrativa. Research, Society and Development, 10(3), e1010312980.

Nunes, S. F. M., Bezerra, D. P. S., Silva, A. P., Lacerda, S. D. L., Silva, I. S., Farias, H. S. L., Silva, T. A., \& Silva, T. K. (2020). Repercussões da síndrome hipertensiva gestacional na saúde mental de gestantes: revisão integrativa da literatura. Brazilian Journal of Development, 6(12), $103995-104006$.

Oliveira, C. A., Lins, C. P., Sá, R. A. M., Netto, H. C., Bornia, R. G., Silva, N. R., \& Junior, J. A. (2006). Síndromes hipertensivas da gestação e repercussões perinatais. Rev. Bras. Saúde Matern. Infant., 6(1), 93-98.

Pereira, G. R., Santos A. A. P., Silva, J. M. O., \& Nagliate, P. C. (2017). Perfil epidemiológico da mortalidade materna por hipertensão: análise situacional de um estado nordestino entre 2004-2013. J. res.: fundam. care. Online, 9(3), 653-658.

Piccolo, D. M. (2018). Qualidade de dados dos sistemas de informação do datasus: Análise crítica da literatura. Ci. Inf. Rev., Maceió, 5(3), 13-19.

Rech, M. S. D., \& David, C. N. (2021). Perfil de gestantes com distúrbios hipertensivos gestacionais e desenvolvimento de instrumento para qualificação do atendimento hospitalar. Research, Society and Development, 10(2), e2701028913. 
Research, Society and Development, v. 10, n. 5, e18610514810, 2021

(CC BY 4.0) | ISSN 2525-3409 | DOI: http://dx.doi.org/10.33448/rsd-v10i5.14810

Ribeiro, V. T., Conselho, T. M. B., Dutra, L. M., Noleto, R. M., Vilasboas, G. T. M., Damini, H. M., Resende, L. A. A., Duarte, I. H., Ferrari, L. L. M., \& Lamounier, L G. A. (2020). Tratamento conservador para rotura hepática em Síndrome HELLP: um relato de caso. Revista Eletrônica Acervo Saúde, 12(10), e5041.

Rodrigues, V. B., Lima, B. B., Fernandes, F. P., \& Elias, D. B. D. (2020). Distúrbios Hipertensivos entre gestantes em um hospital maternidade do município de Aracati-CE. JAPHAC, 7, 155-170.

Silva, G. B. C., \& Cismer, E. D. P. (2017). Mortalidade materna por doença hipertensiva específica da gestação - dheg, maringá - pr, 2010-2014. Uningá Revie, 30(2), 11-16.

Soares, T. C., Santana, L. C. B., Soares, T. C., Ferreira, J. C. S. C., Luz, A. C., Vilarinho, M. F. S. B., Leal, J. D. V., Oliveira, V. A., Almeida, J. R., Araújo, V. S., \& Andrade, R. F. (2018). Fatores de risco relacionados a pré-eclâmpsia: uma revisão integrativa da literatura. Revista Eletrônica Acervo Saúde, Vol.Sup.20 |e437.

Sousa, M. G., Lopes, R. G. C., Rocha, M. L. T. L.F., Lippi, U. G., Costa, E. S., \& Santos, C. M. P. (2020). Epidemiologia da hipertensão arterial em gestantes. einstein (São Paulo), 18, 1-7.

Viellas, E. F., Netto, T. L., Gama, S. G. N., Baldisserotto, M. L., Neto, P. F., Rodrigues, M. R., Martinelli, K. G., \& Domin gues, R. M. S. M. (2021). Assistência ao parto de adolescentes e mulheres em idade materna avançada em maternidades vinculadas à Rede Cegonha. Ciência \& Saúde Coletiva, 26(3), $847-858$. 